Section 8: The role of privately protected

8.1: Networks can be effective mechanisms to represent the interests and concerns of landholders

8.2: Networks can provide support structures for PPA landholders

8.3: Networks can have a role in the monitoring and verification of PPA

8.4: Networks can play an important role in promoting PPA products 8.5: PPA networks should match mission and objectives

8.6: PPA networks, like any organisation, should find ways to support and sustain their activities

Why are PPAs important?

To date, the large majority of recorded protected areas have been created on state-owned lands and waters. Despite these efforts, several ecoregions are poorly represented in the global protected areas network. Many of the world's most important places for biodiversity (and geodiversity) remain outside formal protected areas (Dinerstein et al. 2017) and occur on private, communal or indigenous peoples' land.

Many thousands of PPAs already exist around the world (Bingham et al. 2017), with more being established. But until recently, PPAs have remained a largely hidden resource; they are ignored by some governments, omitted from internationa conservation reporting mechanisms and left out of regional conservation strategies (Stolton et al. 2014). The recognition of existing private conservation efforts, and the establishment of new PPAs, can often fill important gaps in national protected area systems in terms of geographic coverage, ecological representation and protection of endangered habitats and species. Purchas or donation of land and water by individuals can often increase the speed of response to conservation challenges as compared with lengthy government processes of protected area designation (Pasquini et al. 2011). PPAs also bring a wide range of stakeholder into the conservation endeavour, enabling private citizens to contribute directly to process and private entities to cotom-up to the public good.

REFERENCE

Bingham, H., Fitzsimons, J.A., Redford KH. Mitchell, BA, Bezaury - Creel, K.H., Mitchell, B.A., Bezaury - Creel, and Cumming, T.L. (2017). 'Privately protected areas: advances and challenges in guidance, policy and documentation. PARKS 23(1):13-28. AvailCH.2017.PARKS-23-1HB.en

Borrini-Feyerabend, G., Dudley, N., Jaeger, T., Lassen, B., Pathak Broome, N., Phillips, A. and Sandwith, T. (2013). understanding to action. Best Practice Protected Area Guidelines Series no. 20. Gland, Switzerland: IUCN. Available at: https://portals.iucn.org/library/node/29138

Dinerstein, E., Olson, D., Joshi, A., Vynne, C., Burgess, N.D., Wikramanayake, E. Hahn, N., Palminteri, S., Hedao, P. Noss, R., Hansen, M., Locke, H., Ellis, E.C., Jones, B., Barber, C.V., Hayes, R., Kormos, C., Martin, V., Crist, E., Sechrest, W., Price, L., Baillie, J.E.M. Weeden, D., Suckling, K., Davis, C. Sizer, N., Moore, R., Thau, D., Birch, T. Potapov, P., Turubanova, S., Tyukavina, A., De Souza, N., Pintea, L., Brito J.C., Llewellyn, O.A., Miller, A.G., Patzelt, A., Ghazanfar, S.A., Timberlake, J., Kloser, $H$. ., Shennan-Farpón, $Y$ Kindt, R., Lillesø, J-P.B., van Breugel, P., Graudal, L., Voge, M., Al-Shammari, K.F. and Saleem, M. (2017). 'An ecoregion-based approach to protectin half the terrestrial realm?. Bioscience 67:534-545. Available at: https://do org/10.1093/biosci/bix014

Dudley, N. (ed.) (2008). Guidelines for Applying Protected Area Management Categories. Gland, Switzerland: IUCN. Available at: https://doi.org/10.2305/ IUCN.CH.2008.PAPS.2.en

Mitchell, B.A., Stolton, S., Bezaury-Creel, J., Bingham, H.C., Cumming, T.L., Dud ley, N., Fitzsimons, J.A., Malleret-King
D. Redford, K.H. and Solano, P. (2018). Guidelines for privately protected ar eas. Best Practice Protected Area zerland:IUCN xii+ + 200. Gland, Switzerland. IUCN 2 100. 10. Available at: PAG.29.en

Pasquini, L., Fitzsimons, J.A., Cowell, S. Brandon, K. and Wescott, G. (2011). The establishment of large private nature reserves by conservation NGOs: key factors for successful imAvailable at: https://doi.org/10.3017/ S0030605310000876

Stolton, S., Redford, K.H. and Dudley, N. (2014). The Futures of Privately Protected Areas. Gland, Switzerland: IUCN. Available at: https://portals.iucn.org/ library/node/4485

Ol:10.32525/PARAQUARIANAT.2018(6):17.28 ISSN 2309-5237
O Del/de los autor/les.
Es con licencia exclusiva a Guyra Paraguay.

Recibido: 27 de agosto de 2018
Aceptado: 18 del noviembre de 2018

INTRODUCCIÓN

Los tapietes habitan Argentina, Bolivi ñandevas. En Argentina, están localizados en dos comunidades de la provincia de Salta: Misión Los Tapietes, ciudad de Tartatamento Rivadavia. Hasta mediados del $\mathrm{s}$ glo XX, los tapietes desplegaban un patrón de asentamiento nomade o semi-nomade, con un sistema de subsistencia basado en la caza, la pesca y la recolección, y una agricultura incipiente. Los recursos vegetadieta. la comenzo la usupacion del tentorio habitado por los tapietes, instalando haciendas y puestos ganaderos. Esto provocó el conficto con los blancos, a la vez que aceleró el contacto. Paralelamente, en Argentina, se incrementaron las oportunidades de trabajo en los ingenios azucareros y ta bacaleros donde muchas etnias del Chaco, y Paraguay, pais donde se autodenomina gal, Departamento San Martin y Misión La Curvita, a orillas del río Pilcomayo, Depar-

ietes de Argentina: documentación interdisciplinaria del léxico relacionado con el

RESUMEN

presente articulo describe el desarrollo de un proyecto interdisciplinario que abordó el estudio del conocimiento de la diversidad botánica
de la región del Chaco salteño entre los tapie-
tes de Argentina, desde las perspectivas de la lingüistica antropológica y la etno-botánica. A partir de los aportes teórico-metodológicos de la investigación colaborativa e interdisciplinari propusimos, por un lado, contribuir a la documentación de la lengua tapiete (tupí-guarani) a través del estudio del léxico etnobotánico y, por el otro, indagar en los diferentes ambitos quieren significación, abordando las funciones que desempeñan en sus vidas. El análisis lingüistico tuvo como objetivo identificar los me canismos de formacion de nombres de plantas del mundo vegetal entre los tapietes. Las plan-s tas nativas constituyen una de las principales nativas, pertenecientes a 22 familias botánicas, utilizadas en 115 formas de consumo y preparaciones culinarias. Las familias botánicas de mayor relevancia son las Fabáceas y las Sola
náceas, cuyos usos se vinculan principalmo con la alimentación y la medicina, y, en men medida, con actividades relacionadas al ámbito doméstico y al de los combustibles.

PALABRAS CLAVES: lingǘrstica antropológica, etnobotánica, región chaqueña, vocabulario

con ellas los tapietes, se trasladaron para realizar trabajos temporarios (Hirsch 2006). l permanente contacto con la población culturación que implicó un rápido cambio de modo de vida y la adquisición de nuevas prácticas culturales, como la incorporación de nuevos productos alimenticios (Carvajal 1998, Hirsch 2006). En 1983, con el adveniEstado Nacional les reconoció el derecho e propiedad sobre su territorio. En Tartagal, se desencadeno un proceso de urbanzaciôn queimplicó la reducción del espacio que habitaban a cinco manzanas, una de las cuales destinada a la escuela, y la asigños más tarde, en Misión Los Tapietes se construyeron viviendas de material yse instaló agua corriente y electricidad.

Durante la década del 90 compañías petroleras se establecieron en los alrededores de Tartagal. A medida que la ciudad se desarrollaba económicamente, se constru-
ABSTRAC

article describes the development of an in odisciplinary project that addressed the study the Chaco region of Salta among the Tapietes of ical linguistics and ethnobotany. Through theretical and methodological collaborative and aldocumentation we contribute tic and cullurtation of the Tapiete language (Tupi-Guaran of ethnobotanical vocabulary plants octigate the cultural areas within which ins they perform in their lives. The objective of

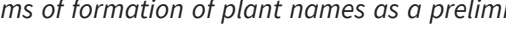
the taxonomic classification of the plants among sapietes. Native plants are one of the main sources of food. We identified 53 native species The most important botanical families are the Fabaceas and the Solanaceas, whose uses the mainly linked to food and medicine, and, to a sphere and to fuels.

KEYWORDS: anthropological linguistics, ethno, Chaco region, ethnobotanical naming.

yeron barrios y caminos, lo que condujo a la destrucción parcial del monte que hasta mediados del siglo XX rodeaba la ciudad. Misión Los Tapietes quedó reducida a un pequeño barrio de aproximadamente 750 personas en la zona periurbana de Tartaga (a)

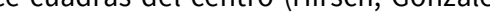
Ciccone 2006). En la actualidad, esta comunidad posee, además, 289 hectáreas, lugr que llaman La Colonia, donde cultivan, principalmente, zapalitios, sandias y maiz para consumo propioy para laventa. Todas las famillas de la comunidad tienen deretoda a explotación det terreno, aunque no 2003).

Una situación marcadamente diferente se observa en Misión La Curvita, pequeño poblado muliétnico de aproximadamente pobo habitantes donde viven seis familias tapietes, todas ellas exogamicas, junto con indigenas tobas, wichies y chorotes. 
LA LENGUA TAPIETE

En Argentina, la lengua tapiete (tupi-guaraní (TG)) se encuentra en serio peligro de extinción. El abandono del nomadismo y la paulatina sedentarización en un contexto semi-urbano, el reemplazo de una economía basada en la caza y la recolección por una economía dependiente de la sociedad criolla y la adopción del español como vehículo de movilidad social ascendente son factores que, entre otros, incidieron en el corte abrupto de la transmisión intergeneracional de la lengua y de los conocimientos tradicionales (Hirsch, González $y$ Ciccone 2006). Ciccone (2015) describe y analiza esta situación que se refleja, particularmente, en la comunidad tapiete de Tartagal, donde se observa la práctica frecuente y extendida de cambio de código entre el tapiete y el español. Junto con la continua retracción de contextos de uso de la lengua, se evidencia entre los hablantes adultos y jóvenes un empobrecimiento del léxico relacionado con la botánica y la zoología, explicable, entre otras cosas, por las transformaciones ecológicas con sus consecuentes transformaciones culturales. En Misión La Curvita observamos un mayor mantenimiento de los modos de vida y de subsistencia característicos de los indígenas chaqueños, aunque también se evidencia un proceso de pérdida de la lengua por parte de las generaciones más jóvenes. Estas razones motivaron la recolección de fitónimos y de textos que hablan sobre los antiguos modos de subsistencia en la región chaqueña.

\section{Antecedentes en la}

documentación lingüística y

etnobotánica

El trabajo de descripción del tapiete comenzó en 1998 y los resultados se viero plasmados en la primera gramática descriptiva de la lengua (Gonzalez 2005). Hasta entonces, no existian registros escritos, con excepción de una lista de alrededor de 600 palabras (Schmidt 1938). A partir de 2002, se consolido un equipo conformado por la antropologa Silvia Hirsch y las lingüistas lorencia Ciccone y Hebe González, quienes realizamos trabajo de documentación 1 Una situación marcadamente diferente es la que
se observa en las comunidades tapietes de Boliviay se observa en las comunidades tapietes de Boliviva
Paraguay, donde la lengua se mantiene en las generaciones más jóvenes, usándose para la comunica-
ción diaria dentro del espacio familiar y público. lingüistica y etnográfica en la comunidad de los saberes tradicionales relacionados de Tartagal. Mas tarde, se sumó la bióloga con la taxonomía botánica y con el uso de sistematización de gran parte de los datos un contexto histórico, ecológico, tecnóó etnobotánicos. Contamos, además, con co y sociocultural del pueblo tapiete de Ardos miembros de la comunidad tapiete de gentina Nuestra investigación no produjo Tartagal, Romualdo Awara Montes y Her- impacto ambiental ni disturbio en produjo melinda Romero, como asistentes perma- versidad vegetal de las zonas entudiadas y nentes, y de otros que colaboraron en la lasidad vegetal de las zonas estudiadas y medida de sus conocimientos, intereses $y$ das patrimonio cultural de los tapietes. posibilidades.

Documentación

interdisciplinaria do de documentación lingüistica a lo largo Mal y zonas aledañas y en Misión de Tartade varios años, los tapietes principalmente Estas tareas se realizaron en distintas épode Tartagal construyeron un proceso de re- cas del año, con el fin de relevar adecuada fexion metalingüística sobre los contextos mente las diferentes fases fenológicas de de uso de la lengua. Los más jóvenes advir- las plantas, y los principales eventos sociotieron la perdida de vocabulario relaciona- culturales ligados a su empleo. Junto con la dos con las prácticas culturales de sus pa- bióloga C. Montani, realizamos recorridas dres y abuelos, y con áreas específicas del con hablantes tapietes por diferentes amconocimiento como la fauna y la flora del bientes donde habitualmente desarrollan ugar. Así, surgió la necesidad de sistemati- actividades como la pesca, la recolección zar y completar la información léxica reco- de alimentos o el cuidado de cultivos y parida en sucesivos trabajos de campo, para ticipamos en actividades cotidianas que redactar un vocabulario de plantas que no involucran el uso de vegetales como, por solo presentara una nómina de especies ejemplo, la recolección del algarrobo y de otanicas, sino que, ademas, brindara in- maiz, la recolección de miel, la preparación formación sobre el significado de los vege- de diferentes alimentos, asi como la elabo-

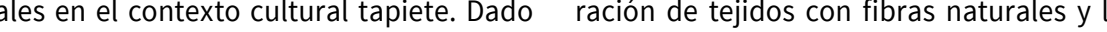

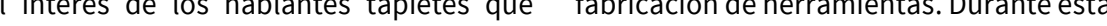
participaronde la recopilacion de palabras, salidas recolectamos 264 ejemplares des argio la necesidad de un trabajo interdis- herbario de las especies sobre las cuales los iplinario aue garantizara la exactitud de hablantes indicaron un nombre en tapietes los datos recogidos, enriquecièndolos por y/o reportaron algún uso. Cuando estas medio del enfoque ennobotánico (Hirsch, recorridas no fueron posibles, trabajamos Gonzalez y Ciccone 2006). Especificamente, con material fresco de las especies recoleeeste proyecto de documentaciốn del lexi- $\quad$ tadas. ${ }^{2}$ También, visitamos los sembradíos co etnobotanico tuvo dos objetivos princi- que los tapietes mantienen en La Colonía pales:

Recabar y sistematizar información sobre el mundo vegetal, centrandonos en la descripción de los usos que sus ancestros hacian de las plantas.

Promover el uso y fortalecer las estrategias de revitalización de la lengua tapiete, a través de la elaboracion de un vocabulario bilingüe de nombres de plantas, ademas dem terial didáctico y de difusión. propusimos contribuir a la revalorizació

Las entrevistas tuvieron como objetivos, porun lado, identificar las especies recolectacer en re os fue determinado taxonónicmicamente en la lunidad $30 \%$ de ellos fue determinado taxonómicamen a supervisión del Dr. Antonio Dal. Montani bajo colaboración del Ing. Ula Karlin yla Lic. Graciela Dis Bisutti. Todos los ejemplares fueron acondicionado y depositados en el herbario del INSEMI) (Instituto de
Investigación y Desarrollo Agroindustrial Horticola
Semillero). ámbitos de la cultura donde estas plantas adquieren importancia. ${ }^{3}$ Estas entrevistas fueron transcriptas y los datos etnobotáncos sistematizados, consignando para cada especie botánica número de herbario, nomla especie, ambiente en el que se encuentra, parte utilizada, categorías y subcate gorías de uso. Enmarcamos estos datos en una breve descripción del contexto cultura de uso. Parte del trabajo de relevamiento de los nombres de plantasy sobre todo, análisis morfológico de fitónimos se a cabo, principalmente, en las casas de comunidad, donde realizamos sesiones $d$ de trabajo, individuales y grupales para corroborar y corregir los datos obtenidos en las sesiones individuales. La Figura 1 ilustra una sesión de trabajo lingüistico.

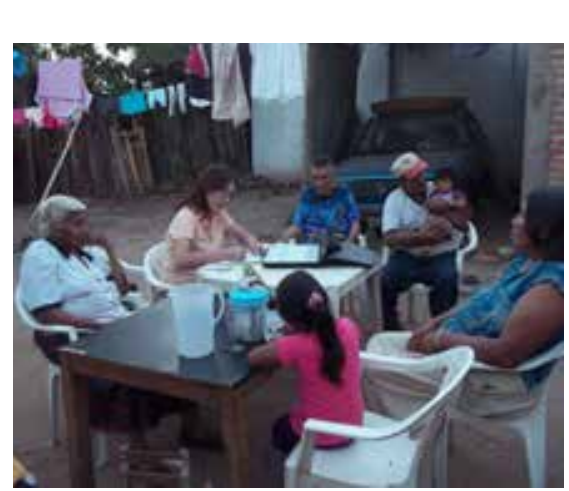
dad tapiete de Tartagal

Durante el desarrollo de la investigacion 作 formación obtenida de manera individua (transcripciôn fonética y ortográfica de los fitonimos, y usos asignados a las plantas) y tomar decisiones acerca del material a producir (libro, láminas, material didáctico). Estos hablantes proporcionaron nombres de plantas, produjeron textos en tapiete y en español con información botanica. güisticas específicas de los fitónimos.

3 Para el relevamiento etnobotánico, realizamos alrededor de 80 entrevistas en profundidad a 23
miembros del a comunidad de entre 30 y 70 años con mayor participación de hombres (65\%) que de bre del consultante, nombre vernáculo de histórica y cultural y, en algunos caso reflexionaron sobre las caracteristicas

Documentación lingüística

Con el fin de contribuir a dilucidar los principios que subyacen a la clasificación taxonómica del mundo vegetal entre los tapietes González (2011) describe los principales mecanismos de formación de fitón mos que se presentan a continuación.

\section{Descripción lingüistica de los fitónimos}

González (2011) distingue entre los fitónimos que primarios, y aquellos que constituyen nombres metafóricos-descriptivos. Los primeros no pueden descomponerse morfológicamente y poseen como único referente la tayi (Handroanthus spp.) es morfológicamente indivisible y su función es designar el 'lapacho'. Varios fitónimos literales pertenecen al fondo léxico de las lenguas TG:
awati 'maíz' (Zea mays), penti 'tabaco' (Nicotiana tabacum), karanda'i 'palmera' (Copernicia alba), entre otros.

Tabla 1. Estructuras morfológicas de los fitónimos

\begin{tabular}{|c|c|c|c|}
\hline $\begin{array}{l}\text { Estructura } \\
\text { morfológica }\end{array}$ & Tapiete & Español & Nombre científico \\
\hline Aumentativo -wasu & $\begin{array}{l}\text { ñtwasü'i'-wasu } \\
\text { tala-AUM }\end{array}$ & Lit. 'tala grande' & Celtis chichape \\
\hline Diminutivo-mí & $\begin{array}{l}\text { ñiwasü'T-mí } \\
\text { tala-AUm }\end{array}$ & Lit. 'tala pequeña' & Celtisiguanaea \\
\hline Construcción posesiva & 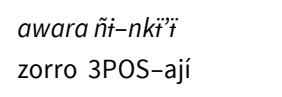 & $\begin{array}{l}\text { ají de monte } \\
\text { Lit. 'aji del zorro' }\end{array}$ & $\begin{array}{l}\text { Capsicum cha- } \\
\text { coense }\end{array}$ \\
\hline Construcción inactiva & $\begin{array}{l}\text { wira puku } \\
\text { palo largo }\end{array}$ & $\begin{array}{l}\text { bobo } \\
\text { Lit. 'palo largo' }\end{array}$ & Tessaria integrifolic \\
\hline Nominalización & $\begin{array}{l}\text { karasiro hú'ü-wa } \\
\text { tártago negro-NOM }\end{array}$ & $\begin{array}{l}\text { Lit. 'tártago (que es) } \\
\text { negro' }\end{array}$ & Ricinus communis \\
\hline Reduplicación & $\begin{array}{l}\text { ha-ya-yási-a } \\
\text { agrio-RED-agrio-NOM }\end{array}$ & $\begin{array}{l}\text { tunilla } \\
\text { Lit. '(que es) muy agrio' }\end{array}$ & $\begin{array}{l}\text { Opuntia elata var } \\
\text { cardiosperma }\end{array}$ \\
\hline Préstamos & kandavare & carnaval & Senna spectabilis \\
\hline Neologismos & $\begin{array}{l}\text { närä'ö-wansu } \\
\text { naranja-AUM }\end{array}$ & $\begin{array}{l}\text { pomelo } \\
\text { Lit.' 'naranja grande' }\end{array}$ & Citrus paradisi \\
\hline
\end{tabular}

Por su parte, los nombres metafóricodescriptivos son aquellos que poseen una structura morfológca interna y se disguen de los nombres literales porque, gemás de nombraruna planta tienen otro referente: por ejemplo, el nombre iwopem (Prosopis aff elata) está compuesto por lexema primario iwope 'fruta del algarrobo' el sufijo diminutivo -mi. Esta palabra significa, por un lado, 'frutita del algarrobo fruta pequeña del algarrobo' y, por el otro, es una etiqueta que designa una planta especifica y diferente a la fruta del algarrobo el 'algarrobillo' (Prosopis aff elata) (Gonzáez 2017:26).

A partir de la estructura morfológica de los fitónimos, identificamos seis tipos de fitónimos metafórico-descriptivos que se lustran en la Tabla 1. La lista se completa que designan especies vegetales tomadas del mundo criollo.

Si bien no podríamos establecer, aún una relación entre la estructura lingüistica

4 Abreviaturass: 1 'primera persona', ' 'tercera per-
sona', AC 'activo', AUM 'aumentativo', C'consonante, CAUS1 ' causativo de verbos estativose intransititativo', EUT 'futuro', INC 'inclusivo', LOC 'locativo', NNEG 'nominalizador negativo', NOM 'nominaliza-'
dor', PL 'plural', POS 'posesivo', PRO 'pronombre', OBL' 'oblicicuo' RED 'reduplicación', RES 'resultativo', 
de los fitónimos y la taxonomía vegetal vernácula, algunos ejemplos ilustran esta relael caso de karuru (Amaranthus aff. standleyanus), fitónimo que designa al 'ataco'. Cuando este término se combina ya sea con el sufijo aumentativo -wasu diminutivo -mi, se obtiene karuruwasu (Amaranthus aff. hybridus) que designa una variedad de karuru de porte más grande que la variedad de referencia y karurumi (Amaranthus affmuricatus) que designa una variedad de karuru de porte más pequeño que la variedad de referencia. Esta relació se esquematiza en la Figura 2.

\section{Variedad Variedad Variedad} 2

'karuru karuruwasu karurum

$\begin{array}{lll}\text { (Amaranthus aff. } & \text { (Amaranthus } & \text { (Amaranthus } \\ \text { Standleyanus) } & \text { aff. hybridus) } & \text { aff muricatus) }\end{array}$

Figura 2. Variedades de karuru 'ataco' (González 2017:27)

\begin{tabular}{|c|c|c|}
\hline Tapiete & Español & Nombre científico \\
\hline$k a^{\prime} a$ & monte bajo ${ }^{+}$ & \\
\hline$k \dddot{t} \dddot{t}$ & ají & Capsicum annuum \\
\hline ñambi & yuyo picante & Acmella oppositifolia \\
\hline penti & tabaco & Nicotiana tabácum \\
\hline piri & tipo de pasto & A determinar \\
\hline p̈̈rö & ortiga & Cnidoscolus tubulosus \\
\hline tayi & lapacho & Handroanthus \\
\hline yetr & batata & Ipomoea batatas \\
\hline$y u^{\prime} a$ & mistol & Ziziphus mistol \\
\hline
\end{tabular}

En guaraní, el vocablo ka'a designa la yerba mate (llex paraguayensis).

\section{Tipos de lexemas y su distribución en el}

Otros lexemas primarios se apartan de la estructura canónica de la palabra tapiete, ya El tapiete posee 15 consonantes - $/ \mathrm{p} / \mathrm{s}, \quad$ la palabra -ej. lexemas trisilábicos- o por $\left./ \mathrm{t} /, / \mathrm{k} /, / \mathrm{kw} /, / \mathrm{s} /<^{\prime}\right\rangle, / \mathrm{mb} /, / \mathrm{nd} /, / \mathrm{ng} /, / / \mathrm{s} /, / \mathrm{s} / \quad$ el tipo de estructura silábica -ej. CVV- que $<\mathrm{sh}>, / \mathrm{h} /, / \mathrm{ts} /<\mathrm{ch}>, / \mathrm{d} 3 /<\mathrm{s}>, / \mathrm{s} /<\mathrm{r}>, / \mathrm{w} /-\mathrm{y}$ despliegan. En principio, estos lexemas no

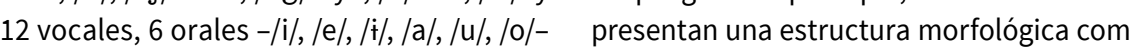
y 6 nasales - $/ \mathrm{i} /$, |ë/, /ïl, /ä/, / /ü/, /ö/-. Como pleja y, al igual que el conjunto anterior, pootras lenguas guaranies que se hablan al seen cognados en otras lenguas $\mathrm{TG}$, como e este de Bolivia -ej. sirionó- y en el norte de avá-guaraní o el guaraní. Este conjunto de Argentina -ej. avá-guaraní (chiriguano)-, el fitónimos se presentan en la Tabla 3. acento recae sobre la penúltima sílaba. La

Suele suceder que el término sin sufijo lengua sólo admite silabas abiertas $-\mathrm{V}, \mathrm{CV}$, Una innovación que parece ser propia diminutivo o sufijo aumentativo es el que CVV- siendo el tipo más frecuente CV. La del tapiete es el uso de dos fitonimos para designa una categoría que incluye a todas gran mayoría de las palabras son disilábi- nombrar, por un lado, el algarrobo blanco las variedades de una planta. Por ejemplo, cas y el contorno silábico más frecuente es wape'i y, por el otro, su fruto iwope. La Tael nombre ñiwasü'i es el término general CV.CV (ej.ta.ta 'fuego'). Dicho de otro modo, bla 4 presenta los cognados de la palabra que hace referencia a todas las variedades la palabra prototipica tapiete consta de dos para designar el algarrobo blanco en avade 'tala', dentro de las cuales se distinguen silabas compuestas, cada una, de una con- guarani, guarani y tapiete, junto con el sigñiwasü'iwasu (Celtis chichape) que es una sonante y una vocal. nificado y los nombres cientificos asignados, según las fuentes consultadas. En ella (Celtis iguanaea) que constituye otra va- Estos rasgos caracterizan una serie de se aprecia la semejanza formal del fitónimo riedad. Esta relación se esquematiza en la lexemas primarios, con cognados en otras en la tres lenguas con leves diferencias seFigura 3.

lenguas TG cercanas al tapiete como el avá- mánticas.

guarani y el guarani, que se enlistan en la Tabla 2.

\section{Especie}

ñiwasü'i' 'cualquier tipo de tala'

Variedad 1

ñtwasü'ïwasu 'tala'

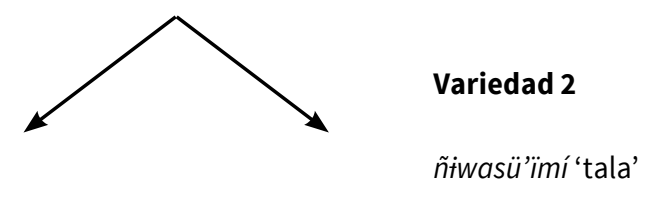

(Celtis chichape)
Tabla 3. Fitónimos trisilábicos de origen TG

\begin{tabular}{ccc}
\hline Tapiete & Español & Nombre cientifico \\
\hline andai & calabaza & Cucurbita moschata \\
awati & maíz & Zea mays \\
iwope & fruta del algarrobo blanco & \\
kapi'i & césped & Panicum trichanthum \\
karanda't & palmera & Copernicia alba \\
karasiro & tártago & Ricinus communis \\
kawata & cháguar & Bromelia hieronymi \\
kimanda & poroto & Phaseolus spp \\
kimbaru & chañar & Geoffroea decorticans \\
mandityu & algodón & Gassypium spp. \\
mandi'o & mandioca & Manihot esculenta \\
ñandipa & hediondilla & Cestrum parqui \\
uruku & urucú & Bixa orellana \\
wape't & algarrobo blanco & Prosopis alba \\
\hline
\end{tabular}

Tabla 4. Denominaciones del algarrobo blanco en tapiete, guaraní y avá-guaran

\begin{tabular}{|c|c|c|c|}
\hline & Cognado & Significado & Nombre científico \\
\hline \multirow[b]{2}{*}{ Ava-Guaraní } & igwope & algarroba (Dietrich 1986:356) & No consigna \\
\hline & Fvopei & 'algarrobo blanco, 'árbol de las mezquitas' & $\begin{array}{l}\text { Prosopis juliflora Griseb; } \\
\text { Prosopis alba Griseb } \\
\text { (Hart et al. 1996:56) }\end{array}$ \\
\hline Guaraní & ivopéî & $\begin{array}{l}\text { 'Nombre común a varias especies de Prosopis, abundan- } \\
\text { tes en el Chaco' (Gatti 1985:154) }\end{array}$ & \\
\hline \multirow{2}{*}{ Tapiete } & iwope & Fruta del algarrobo blanco & \\
\hline & wape’t & Algarrobo blanco & Prosopis alba Griseb. \\
\hline
\end{tabular}

* Hart et al. (1996) presentan las denominaciones 'algarrobo blanco' y'árbol de las mezal
consignan, estos no tienen una correspondencia biunivoca con los fitónimos en español.

En la Tabla 4 se puede observar que el plejidad morfológica y transparencia se- c. (j)wïrä=penti 'palo amarillo' tapiete ha desarrollado dos formas a partir mantica emergen claramente, como en (1), de ivopei (avá-guarani) e îvopêi (guaraní): el donde el fitónimo, que designa un tipo de d. (i)wira=pinta 'patapata' término para designar la fruta del algarro- hongo, está formado a partir de una cons- Ximenia americana bo se forma a partir de las primeras tres si- trucción posesiva 'pene del diablo'. labas de la palabra -ej. tivopet $\rightarrow$ iwope-; en tanto que el têrmino para designar el árbol $\quad$ 1) a. ña=y-a.cha 'tipo de hongo' se forma a partir de las tres últimas silabas diablo=3POS-pene - ej. . $i v o p e t \rightarrow$ wape't.$-^{5}$

Algunas palabras sugieren la existencia Existen fitónimos cuyo contorno silábi- de restricciones ligadas al contorno silabico se aparta de la estructura canonica de co y al número de silabas admitidas como la lengua. Estos lexemas despliegan tres 0 se reflejan en fitonimos formados por comcuatro silabas, siendo el tipo (C)V.CV=CV.CV. puestos cuyo primer miembro es la palabra A menudo, estos fitonimos constituyen le- FWira 'palo, arbol', lexema trisilabico que xemas metaforico-descriptivos, cuya com- pierde la primera vocal con el objeto de 5 De la tabla 4 se desprende, ademas, que la forma pestablecer la cadena sibica no marcada. el avà-guarani es semejante a la forma tapiete Algunos ejemplos se presentan en (2). ${ }^{6}$ iwope; en tanto, la forma ivopei que presentan Har $\begin{array}{ll}\text { et al. (1996:56) para la misma lengua se asemeja } & \text { 2) a. (ił)wira=wewi 'palán palán' } \\ \text { a îvopế termino documentado por Gatti para el } & \text { Nicotiana glauca }\end{array}$ guarani (1985:154). Esta leve diferencia formal en avá-guarani se explica por las variedades que documentan los autores: Dietrich (1986) present
datos relevados entre los ava-auarani de Tartagal,
Argentina;

b. (i)wira=i-piro 'guayacán' Argentina; en tanto Hart et al. hacen lo propio con
datos provenientes de las provincias de O'Connor y Gran Chaco, del Departamento de Tarija, Bolivia
$(1996 ; 3)$.

b. (t) Wira=i-piro 'guayacán' Caesalpinia paraguariensis

6 La forma apocopada de iwira 'palo' resulta en creación de un homónimo de la palabra wira cuy
significado es 'pajaror'.
En esta sección hemos querido ilustrar la complejidad de la tarea de identificar certeramente la naturaleza del fitónimo como lexema metaforico-descriptivo y, en conproporción relativa de lexemas primarios -descriptivo, dentro del léxico

Con el fin de producir el vocabulario b lingüe, creamos una base de datos léxica en la cual se almaceno la información linguística, etnobotánica y etnograficca asociada a cada planta. La información botanica incluyo el nombre cientifico, la familia botánica y, en algunos casos, la descripción botánica. La información etnografica abordó los usos culinarios y medicinales de las plantas y otros relacionados con el ámbito do- 
méstico y las actividades artesanales. Así, hama awati tánta-ma o-ho-rà ealiza yos un resces maíz duro-RES 3AC-ir-SUB fitónimos que incluyó sinónimos, así como entonces cuando el maiz se iba usos onomísticos $y$ toponímicos, A partir endureciendo,

221 especie relevadas, 25 préstamos, más CAUS1-secar-SUB todo guardar-PL

221 especie relevadas, 25 préstamos, más cinco especies para las 25 presstos hos, nantes formar su nombre tapiete.

\section{Los textos}

Para contextualizar etnográficamente los datos léxicos, recogimos textos relacionados con el uso de las plantas que
fueron transcriptos, interlinearizados -es decir, traducidos morfema por morfemay traducidos al español. Aquí presentamos como ejemplo un texto sobre antiguos hacomo ejemplo un texto sobre antiguos haria Mercado, hablante tapiete de Misión Los Tapiete.

\section{Las plantas que comian antiguamente}

a-mbe'u-po-yi-mba arika' SG.AC-narrar-FUT-FRC-EVD antes voy a contar antes lo de antes arika'e-wa-re ñánde-re hou-wa tos-NOM-PL nosotros (INC)-PL 3:comer-NOM lo que comían nuestros antiguos paisanos

ñin-kisemba-pe o-ï-mba hou arika'e 3POS-cerco-LOC 3AC-estar-EVD 3:Comer an logne tenich 3:comer-EVD antes anco 3:comer-EVD antes
comían antes anco,

awati, yeti, mindaka, ndakara maíz batata zapallo noroto maiz, batata, zapallo, poroto

awati tánta-mba awati piriri maíz duro-NNEG maíz chispas maiz blando, pochoclo

ha'e ä karai-re

ra hombre blanco-PL a eso ahora los criollos

pochoklo hei shu awati piriri pochoclo 3:decir 3:PRO.OBL maíz chispas ha'e-mba hou artka'e artka'e-wa-re
el/ella-EVD 3:comer antes antes-NOM-P so comían antes los de antes
22 familias botánicas, utilizadas en 115 formas de consumo y preparaciones cullnarias. Las familias botánicas de mayor relevancia son las Fabáceasy las Solanáceas, cuyos usos se vinculan principalmente con la alimentación y la medicina y en menor medida, con actividades relacionadas ámbito doméstico y al de los combustibles. Por los volúmenes aprovechados, la variedad de formas en las que son consumidos y la importancia que poseen en su dieta destacan ciertas especies que se presentan en la Tabla 2

Como otros grupos chaqueños, antiguavegetales alimenticias principalmente forma de alimentos crudos, siendo el fruto la parte vegetal más frecuentemente consumida como piscolabis por niños y adultos en sus recorridas por el monte. Los fruto de algunas plantas -iwopemi 'algarrobillo' (Prosopis aff. elata), iwope tai 'algarrobo negro' (Prosopis nigra), kimbaru 'chañar' (Geoffroea decorticans), wape't 'algarrobo blanco' (Prosopis alba), yu'a 'fruto de mistol' (Sarcomphalus mistol), wampër 'tusca' (Acacia aroma)- son recolectado en gran cantidad para ser desecados al so y utilizados en la preparación de añapa bebida a base de maíz, algarroba o misto molido mezclado con agua que se bebe antes de que fermente-, harinas, panificados y bolanchaos -galletas de harina de chañar, algarrobillo y algarrobo negro. Los tapietes de La Curvita aún hoy mantienen este há bito, asi como es frecuente que consuma otros alimentos crudos en forma de ensal das. En tanto, los tapietes de Tartagal han incorporado a sus dietas frutos de especies exoticas tales como el mango, la guayaba y la banana (Montani y Scarpa 2016).

\section{Medicina}

Identificamos 51 especies vegetales permicas que los tapietes utilizan con fines medicinales, ya sea como antitérmico o bien para tratar afecciones digestivas, trastornos de la pie $y$, en menor medida, odontalgias, afecciones del sistema reproductor y del sistema respiratorio. Algunas plantas también intervienen en rituales curativos.

Las principales afecciones tratadas con remedios vegetales son el dolor de estomago y el dolor de higado o tipi'a rast que con el consumo de
Tabla 5. Principales especies relacionadas con la alimentación

\begin{tabular}{|c|c|c|c|}
\hline Tapiete & Español & Nombre científico & Observaciones \\
\hline wape't & algarrobo blanco & Proposis alba & $\begin{array}{l}\text { Consumen el fruto fresco y la harina. Mujeres y niños suelen chupar la pulpa de } \\
\text { los frutos frescos maduros. Se prepara como añapa sólo o mezclado con yu'a } \\
\text { 'fruta de mistol' o con la frutata del iwope tai 'algarrobo negro'. }\end{array}$ \\
\hline kimbaru & chañar & Geoffroea decorticans & $\begin{array}{l}\text { Sus frutos disecados se hierven y se muelen. Con la harina elaboran galletas } \\
\text { kinta que consumen con grasa de pescado o acompañando sopas de pescado } \\
\text { o de vizcacha. }\end{array}$ \\
\hline iwowi & bola verde & Capparis speciosa & Sus frutos se consumen hervidos y acompañados con grasa de pescado. \\
\hline ñambiwasu & yuyo picante & Acmella opositifolia & \\
\hline awara ñiktït & aji de monte & Capsicum chacoense & Condimentos o aromatizantes, utilizados en ensaladas y sopas. \\
\hline kipt & aji & Capsicum chacoense & \\
\hline wawasu & sacha sandía & Capparis salicifolia & Sus frutos en estado inmaduro son muy tóxicos. \\
\hline wawasuna & sacha membrillo & Capparis tweediana & Se consume del árbol, luego de haber descartado la cáscara y las semillas. \\
\hline yu'a & fruto del mistol & Ziziphus mistol & Se consume como añapa o harina, a veces mezclado con vainas de algarrobo. \\
\hline
\end{tabular}

Tabla 6. Plantas utilizadas para afecciones del sistema digestivo

\begin{tabular}{|c|c|c|c|}
\hline & Tapiete & Español & Nombre científico \\
\hline \multirow{14}{*}{ Sistema digestivo } & wampërë & tusca & Acacia aroma \\
\hline & tanimbu & huáncar, coronillo blanco & $\begin{array}{l}\text { Bougainvillea campanulata } \\
\text { Bougainvillea stipitata }\end{array}$ \\
\hline & wapaya & papaya & Carica papaya \\
\hline & 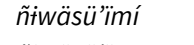 & tala & Celtis iguanaea \\
\hline & ñtwäsü̈'iwasu & tala & Celtis chichape \\
\hline & tiwasu inku & brea & Cercidium praecox \\
\hline & kapi'i pishea & cedrón & Cymbopogon spp. \\
\hline & paiko & paico & Disphania ambrosioides \\
\hline & $\begin{array}{l}\text { tipeisha } \\
\text { pungapewa }\end{array}$ & coca de monte & $\begin{array}{l}\text { Erytrhoxylum argentinum } \\
\text { Heliotropium procumbens }\end{array}$ \\
\hline & saini & tuna de casa & opuntia ficus-indica. \\
\hline & 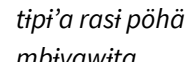 & $\begin{array}{l}\text { Lit. 'remedio para el dolor de higado' } \\
\text { duraznillo }\end{array}$ & $\begin{array}{l}\text { Pluchea sp. } \\
\text { coltatsifor }\end{array}$ \\
\hline & $\begin{array}{l}\text { mbiyawita } \\
\text { totia }\end{array}$ & $\begin{array}{l}\text { duraznillo } \\
\text { tomatillo }\end{array}$ & $\begin{array}{l}\text { Salta triflora } \\
\text { Solanum sisymbriifolium }\end{array}$ \\
\hline & toti'amí & & Solanum aridum \\
\hline & & ancoche & Vallesia glabra \\
\hline
\end{tabular}

comidas pesadas, la ingesta de alcohol y tukuru, micosis cutánea o tipiritt y diviesos también, el uso de raices, tales como el shi piwasu 'calabuchi' (Petiveria alliaceae) y e chacoense). La ingesta por via oral de la -fruto, hojas y parte aèrea- y se aplican, toti'a tomatilo' (Solanum sisymbriifolium) decocción de las hojas de las especies uti- generam lizadas es la forma más frecuente de prepa- afección corporal. La Tabla 4 presenta las presenta las principales plantas utilizadas ración. La Tabla 3 presenta las plantas utili- plantas utilizadas para tratar estas afeccio- para tratar las afecciones respiratorias. zadas para tratar las afecciones del sistema nes. digestivo.

Otras plantas que intervienen en la cura de Identificamos, además, especies que, problemas del sistema reproductor, el sisteLas plantas se utilizan también como ci- a través de la ingesta oral de la decocción ma circulatorio, reproductor, nervioso, uricatrizantes y madurativos para tratar los de sus hojas, se usan principalmente como nario, osteo-muscular, odontalgias o como trastornos de la piel: quemaduras, sarna o antitusivas y descongestivas. Es frecuente, antitérmicos se presentan en la Tabla 6. 
Tabla 7. Plantas utilizadas para los trastornos de la piel

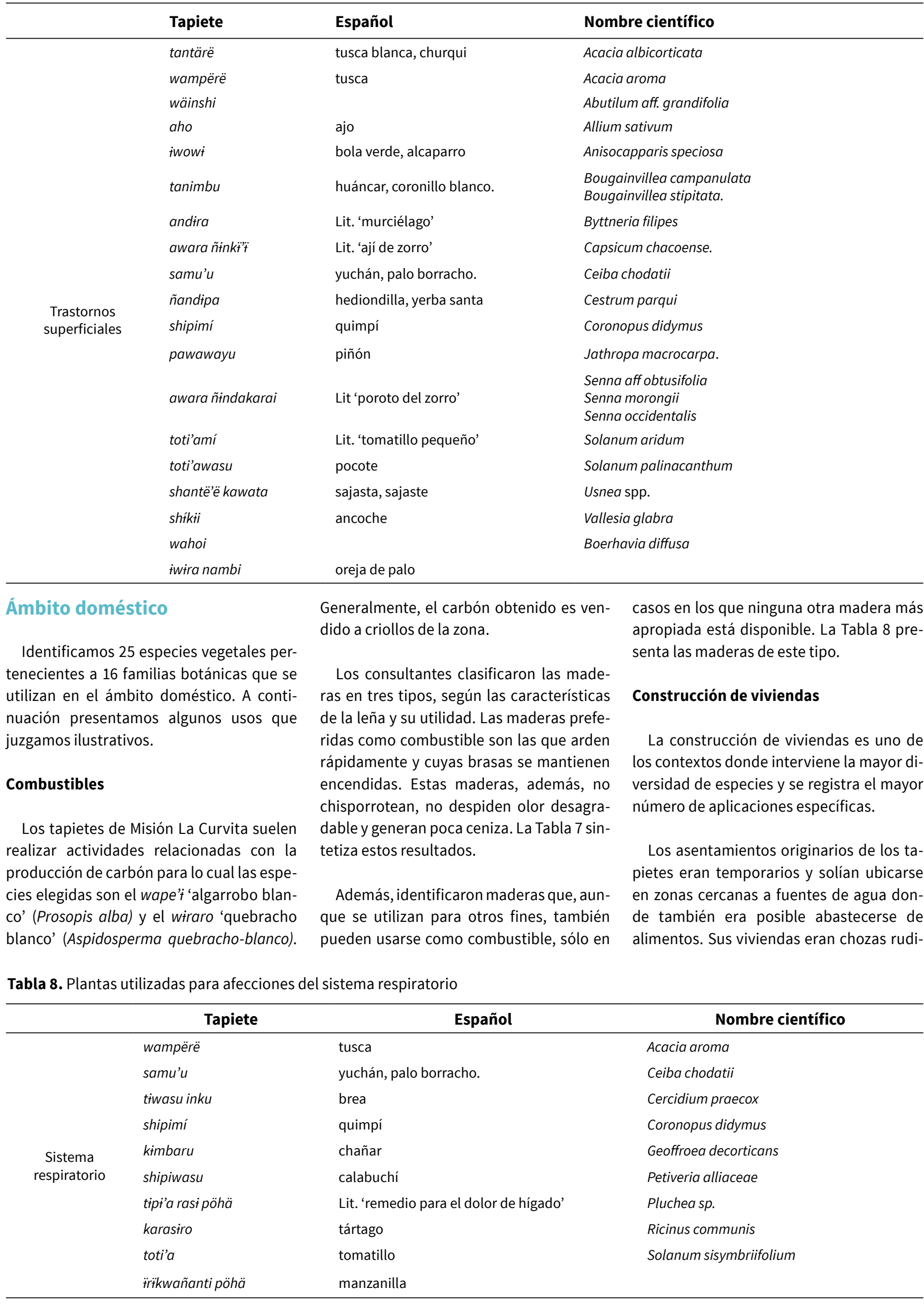

\begin{tabular}{|c|c|c|c|}
\hline & Tapiete & Español & Nombre cientifico \\
\hline Sistema circulatorio & $\begin{array}{l}\text { ñandipa } \\
\text { mburukuyamí }\end{array}$ & $\begin{array}{l}\text { hediondilla, yerba santa } \\
\text { murucuyá, pasionaria }\end{array}$ & $\begin{array}{l}\text { Cestrum parqui } \\
\text { Passiflora mooreana }\end{array}$ \\
\hline Sistema reproductor & $\begin{array}{l}\text { wampërë } \\
\text { ñandipa } \\
\text { andai } \\
\text { samu'u } \\
\text { sanya } \\
\text { iwira nambi }\end{array}$ & $\begin{array}{l}\text { tusca } \\
\text { hediondilla, yerba santa } \\
\text { anco } \\
\text { yuchán, palo borracho } \\
\text { sandia } \\
\text { oreja de palo }\end{array}$ & $\begin{array}{l}\text { Acacia aroma } \\
\text { Cestrum parqui } \\
\text { Cucurbita moschata } \\
\text { Ceiba chodatii } \\
\text { Citrullus lanatus }\end{array}$ \\
\hline Sistema nervioso & $\begin{array}{l}\text { iwowi } \\
\text { ñantirëmi }\end{array}$ & bola verde, alcaparro & $\begin{array}{l}\text { Anisocapparis speciosa } \\
\text { Merremia dissecta }\end{array}$ \\
\hline Sistema urinario & $\begin{array}{l}\text { nimö } \\
\text { shipimí } \\
\text { taył } \\
\text { märä } \\
\text { totia }\end{array}$ & $\begin{array}{l}\text { limón } \\
\text { quimpí } \\
\text { lapacho } \\
\text { vergonzosa } \\
\text { tomatillo }\end{array}$ & $\begin{array}{l}\text { Citrus limon } \\
\text { Coronopus didymus } \\
\text { Handroanthus spp. } \\
\text { Mimosa xanthocentra } \\
\text { Solanum sisymbriffolium }\end{array}$ \\
\hline $\begin{array}{l}\text { Sistema ósteo- } \\
\text { muscular }\end{array}$ & $\begin{array}{l}\text { iwowi } \\
\text { ómpea pöhä } \\
\text { mbiyawitawasu } \\
\text { owai } \\
\text { tapiti iyuki } \\
\text { käwë hohówia }\end{array}$ & $\begin{array}{l}\text { bola verde, alcaparro } \\
\text { Lit. 'remedio para la quebradura' } \\
\text { duraznillo del ciénago } \\
\text { Santa Lucía } \\
\text { Lit. '(el que es) hueso muy verde' }\end{array}$ & $\begin{array}{l}\text { Anisocapparis speciosa } \\
\text { Hemia salicifolia } \\
\text { Coccoloba spinescens } \\
\text { Ruprechtia laxiflora } \\
\text { Commelina erecta }\end{array}$ \\
\hline Antitérmico & $\begin{array}{l}\text { iwowi } \\
\text { taku pöhä } \\
\text { karasiro }\end{array}$ & $\begin{array}{l}\text { bola verde, alcaparro } \\
\text { altamisa } \\
\text { tártago }\end{array}$ & $\begin{array}{l}\text { Anisocapparis speciosa } \\
\text { Parthenium hysterophorus } \\
\text { Ricinus communis }\end{array}$ \\
\hline Odontagias & $\begin{array}{l}\text { samu'u } \\
\text { ka'a tai } \\
\text { tata yiwa }\end{array}$ & $\begin{array}{l}\text { yuchán, palo borracho } \\
\text { barba de chivo } \\
\text { mora, tatajyva }\end{array}$ & $\begin{array}{l}\text { Ceiba chodatiii } \\
\text { Clematis montevidensis } \\
\text { Maclura tinctoria }\end{array}$ \\
\hline $\begin{array}{l}\text { Trastornos } \\
\text { metabólicos }\end{array}$ & $\begin{array}{l}\text { uruku } \\
\text { uru pampe }\end{array}$ & $\begin{array}{l}\text { urucú } \\
\text { uña de gato }\end{array}$ & $\begin{array}{l}\text { Bixa orellana } \\
\text { Macfadyena aff. hassleri }\end{array}$ \\
\hline
\end{tabular}

Tabla 10. Tipos de leña

\begin{tabular}{|c|c|c|c|}
\hline \multicolumn{4}{|c|}{ Maderas utilizadas preferentemente como leñas } \\
\hline Tapiete & Español & Nombre científico & Características \\
\hline wampërë & tusca & Acacia aroma & Buena calidad de brasa y de fuego. \\
\hline tantärë & tusca blanca & Acacia albicorticata & $\begin{array}{l}\text { Debe secarse completamente para ser utilizada como leña. Genera } \\
\text { brasa y ceniza en abundancia. Permanece encendida por un período } \\
\text { prolongado. }\end{array}$ \\
\hline kiripai & cebil & $\begin{array}{l}\text { Anadenanthera } \\
\text { colubrina }\end{array}$ & $\begin{array}{l}\text { Mantiene el fuego encendido y produce poca ceniza. Es una leña } \\
\text { apropiada para cocinar. Para utilizar su corteza como combustible, se } \\
\text { recomienda hacer carbón con ella cuando está verde; de lo contrario, se } \\
\text { convierte en ceniza rápidamente. }\end{array}$ \\
\hline wiraro & quebracho-blanco & Aspidosperma & Produce brasa y carbón en abundancia; no chispea e irradia mucho calor. \\
\hline wiraita & palo santo & Bulnesia sarmientoi & $\begin{array}{l}\text { Arde a pesar de la humedad. Como su resina no se apaga, con su madera } \\
\text { hacian antorchas y fogones. La usaban como comburente para friccionar } \\
\text { el durmiente del taladro o generar la ignición. Su leña, además, era } \\
\text { utilizada para cocinar en la olla. }\end{array}$ \\
\hline wape't & algarrobo blanco & Prosopis alba & $\begin{array}{l}\text { Se utiliza especialmente en las épocas de frío. Aunque no arde rápido, } \\
\text { mantiene el fuego encendido, produce abundante brasa y buen carbón. }\end{array}$ \\
\hline iwope tai & algarrobo negro & Prosopis nigra & $\begin{array}{l}\text { Tiende a producir chispas que se intensifican cuando arde junto con la } \\
\text { leña del algarrobo blanco. }\end{array}$ \\
\hline
\end{tabular}


Tabla 11. Leñas que se utilizan en combinación con otras

\begin{tabular}{|c|c|c|c|}
\hline \multicolumn{4}{|c|}{ Leñas que se utilizan en combinación con otras maderas } \\
\hline Tapiete & Español & Nombre cientifico & Caracteristicas \\
\hline wirai & suncho & Baccharis salicifolia & $\begin{array}{l}\text { Buena para iniciar el fuego porque arde rápido, } \\
\text { aunque no lo mantiene encendido y se consume } \\
\text { rápidamente. La usan para cocinar en la olla. }\end{array}$ \\
\hline wiraipiro & guayacán & Caesalpinia paraguarienses & $\begin{array}{l}\text { Produce buena brasa y se mantiene encendida } \\
\text { por un periodo prolongado. Sin embarago, humea } \\
\text { demasiado y arde poco. La combinan con otras } \\
\text { maderas. Los tallos se utilizan como asador porque } \\
\text { no transfieren sabor amargo a la comida. }\end{array}$ \\
\hline
\end{tabular}

Tabla 12. Tipos de maderas que no se utilizan como combustible

\begin{tabular}{|c|c|c|c|}
\hline \multicolumn{4}{|c|}{ Maderas no utilizadas como leña } \\
\hline Tapiete & Español & Nombre cientifico & Caracteristicas \\
\hline tanimbu & huáncar & $\begin{array}{l}\text { Bougainvillea campanulata } \\
\text { Bougainvilea stipitata }\end{array}$ & $\begin{array}{l}\text { Su ceniza se utiliza como madurativo de } \\
\text { espinas. }\end{array}$ \\
\hline 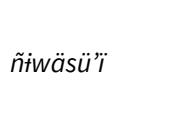 & tala & Celtis spp. & $\begin{array}{l}\text { Con su madera manipulan alimentos debido } \\
\text { a que no transfiere sabor amargo a las } \\
\text { comidas. }\end{array}$ \\
\hline tiwasu inku & brea & Cercidium praecox & $\begin{array}{l}\text { Se consume rápidamente, sin formar brasa, } \\
\text { dejando sólo cenizas. }\end{array}$ \\
\hline wirinde't & urundel & Schinopsis lorentzii & $\begin{array}{l}\text { Aunque la consideran buena para usar como } \\
\text { combustible, no suelen utilizarla porque } \\
\text { chispea mucho. }\end{array}$ \\
\hline yiwa't & mistol & Ziziphus mistol & $\begin{array}{l}\text { Genera una ceniza blancuzca que se utiliza } \\
\text { como lejía para hacer mote pelado. }\end{array}$ \\
\hline
\end{tabular}

mentarias en forma de cúpula, de pequeñas dimensiones que constaban de un solo arion actividades se desarrollaban alrededor de la toldería. Durante la época de cosechade la caña de azúcar, los tapietes vivían en los ingenios donde construín sus viviendas que quemaban cuando culminaba la cosecha. Utilizaban troncos de madera dura pero flexible - wircita 'palo santo' (Bulnesia sarmientoi), tayi 'lapacho' (Tabebuia sp.) y wiraro 'cedro' (Aspidosperma quebracho blanco) - que unían en sus extremos $y$ ataban con cuero de chiva o fibras de samu'u 'yuchán' (Ceiba sp.). Por encima, colocaban diversas gramineas o kapi'i 'pasto', aunque también, por ser derechas y tupidas, solian utilizar ramas de shíkii o pikasu himbiu 'anchoche' (Vallesia glabra), tänkwürér')' 'cañas de azúcar' (Saccharum officinale) y de yakurimbi'u (Solanum argentinum). Cuando las ramas se secaban, o bien luego de un intenso viento o lluvia, debían ser repuestas. Actualmente no construyen más este tipo de chozas.

\section{Huertas y jardines}

Entre los tapietes que residen en zonas urbanas, es frecuente observar cercos de dimensiones pequeñas (en promedio so de $6 \mathrm{~m}$ de largo por $4 \mathrm{~m}$ de ancho) en los que cultivan, de forma entremezclada y para fi-

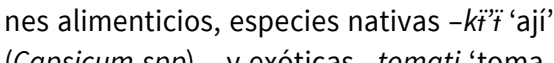
(Capsicum spp) - y exóticas -tomati 'tomate'-, y tambien frutales -wayawa 'guayaba' (Psidium sp.), mango (Mangifera sp) y yiwa' 'mistol' (Ziziphus mistol), entre otras-. Las mujeres mayores de la comunidad suelen cultivar plantas que utilizan para curar aicadura -wawasuna 'sacha membrillo' (Capparis tweediana) - o bien como alimento - (twowt 'bola verde' (Capparis speciosa) $y$, tambien, plantas ornamentales -achira (Canna edulis) y Brugmansia spp.-. En las zonas rurales, en cambio, en sus cercos kisemba, que ubican cerca de sus viviendas, siembran, principalmente, especies nales.

\section{CONCLUSIÓN}

En este artíc mientos teorico-metodológicos de un proyecto cuyo objetivo ha sido contribuir a la documentación de la lengua tapiete a tra- vés del estudio del léxico etnobotánico. Entre los tapietes de Argentina, el proceso de desgaste cultural, sumado al reducido número de hablantes que aun preservan conocimientos relacionados con el uso de las plantas da cuenta de la pérdida de saberes de supervivencia y, en consecuencia, de urgencia del trabajo de documentación lingǘstica y etnográfica. Previsiblemente, los tapietes cuya vida se desarrolla en el ámbto rural-comunidad de Misión La Curvitapreservan más conocimientos relacionados con las plantasy sus usos. Entre los tapietes de Tartagal, por el contrario. Se observa un alto grado de préstamos culturales a través del reemplazo de especies vegetales $y$ del uso que hacen de ellas, lo que refleja la influencia de grupos criollos.

A fin de comparar el conocimiento que los tapietes poseen del mundo vegetal con otros grupos indígenas guaraníticos y chaqueños, resulta imperioso incrementar el trabajo de documentación y archivo con la colaboración de las comunidades. Esto contribuirá tanto al esclarecimiento del origen étnico del pueblo tapiete, como de las relaciones que mantuvieron con otros grupos indigenas de la región, además de contribuir al conocimiento de los sistemas de clasificación nominal y de categorización del Gran Chaco.

AgRADECIMIENTOS

A los hablantes tapietes de las comunidades de Misión Los Tapiete y Misión La Curen ellos, especialmente, a este proyecto y Montes y su madre Helena Cabeza.

Esta investigación ha sido posible gracias al financiamiento de los proyectos PIC 2015-2997 "Léxico tapiete (Tupí - Guarani): mecanismos de formación de palabras y lexicografía." Agencia Nacional de Promoción Cientifica y Tecnológica (ANPCYT); CICITCA 21/F876 y F891 "Conocimiento tapiete (tupi-guarani) de la biodiversidad chaqueña. vocabulario bilingue de plantas: sus usos significaciones" de la Universidad Nacional de San Juan, Argentina.

REFERENCIAS

Arce Birbeuth, E., R. Gutiérrez Condori 1. Gutiérrez Condori y R. López Véliz. 2003. Estrategias de sobrevivencia entre los tapietes del Gran Chaco. La Paz DIEYT. CED. CER-DET. PIEB. 206pp.

Arenas, P. 1995. Guía temática para entrevista etnobotánica con aborígenes del Gran Chaco (Ms).

Arenas, P. 2003. Etnografía y alimentación entre los Toba-Ñachilamole\#tek y Wichí-Lhuku'tas del 'Chaco Central (Argentina), Buenos Aires: El autor. 563pp.

Ayza Arraya, F. 1998. Etnoconocimiento tapiete de los recursos naturales de llanura chaqueña XII Reunión anua de etnología Vol:(I) 333-361. La Paz, Bolivia: MUSEF.

Bates, D. M. 1985. Plant utilization: patterns and prospects. Economic Botany 39(3): 241-265.

Carvajal, S. 1998. Etnohistoria y ocupación espacial del pueblo tapiete. Anales de la Reunión Anual de Etnologia, La Paz. 363-385pp.

Ciccone, F. 2015. Contacto, desplazamiento y cambio linguístico en tapiete (tupí-guarani). Tesis de Doctorado. Facultad de Filosofía y Letras, Universidad de Buenos Aires, Buenos Aires.

Gatti, C. 1985 [1956]. Enciclopedia guarani-castellano de Ciencias Naturales conocimientos paraguayos. Asunción Arte Nuevo Editores. 329pp.

González, G. 1984. Entre los chaneguaranies de Pikuiva, Wendague y Nambyrenda. Revista Sociedad Cientifica del Paraguay, XVIII - № 1 y 2, 149 201.

González, H. A. 2003. Los tapietes: esbozo etnografico y sociolinguistico. In: Fernández Garay, A. y L. Golluscio (Eds.) Temas de Lingüistica Aborigen II. Buenos Aires: UBA. 263-287pp. 
González, H. A. 2005. A grammar of Tapiete (Tupi-Guarani). PhD Dissertation, versity of Pittsburgh, Pittsburgh.

González, H. A. y G. Scarpa. 2008-2010. Conocimiento tapiete (tupi-guaraní) de la biodiversidad chaqueña. Textos y vocabulario bilingüe de plantas: sus usos y significaciones. Proyecto CICITCA $21 /$ F876. San Juan: Consejo de Investigación Científica, Técnica y de Creagión Airtística (CICITCA), Secretaría de Ciónciay yécnica de la UNS.

González, H. A. 2011. Léxico etnobotánico tapiete (tupí-guaraní), lengua del Chaco argentino. Indiana, 28, 255-288.

González, H. A. (Ed.). 2017. Ecos del mundo vegetal entre los tapietes de Argenti-
na. Diccionario etnobotánico Vol:(64). Muenchen: LINCOM. 187pp.

Hart, T., R. Cuba y A. Turner. 1996. Plantas útiles de la zona del Itika Guasu. Tarija, Bolivia: Centro de Estudios Regionales para el Desarrollo de Tarija. 130pp.

Hirsch, S. M. 2006. El pueblo tapiete de Argentina: historia y cultura. Buenos Aires: Instituto de Lingüistica. Facultad de Filosofía y Letras. Universidad de Buenos Aires. 143pp.

Hirsch, S. M., H. González y F. Ciccone. 2006. Lengua e identidad: ideol lingüísticas, pérdida y revitalización dela lengua entre los tapietes. Indion 23:103-138.

Montani, M. C. y G. F. Scarpa. 2016 Recursos vegetales y practicas alimentarias
entre indigenas tapietes del noreste de la provincia de Salta Arsentina Darwiniana, nueva serie 4(1):12-30.

Scarpa, G. 2007. Hacia una taxonomía vegetal chorote I: fitonimia, sistema nomenclatural y comparación dialectal. 119

Schmidt, M. 1938. Los Tapietés. Revista de la sociedad científica del Paraguay $4(2), 28-63$.
Catacantha ferruginea (Draudt, 1929) [Insecta, Lepidoptera, Saturniidae], una nueva adición a la entomofauna paraguaya

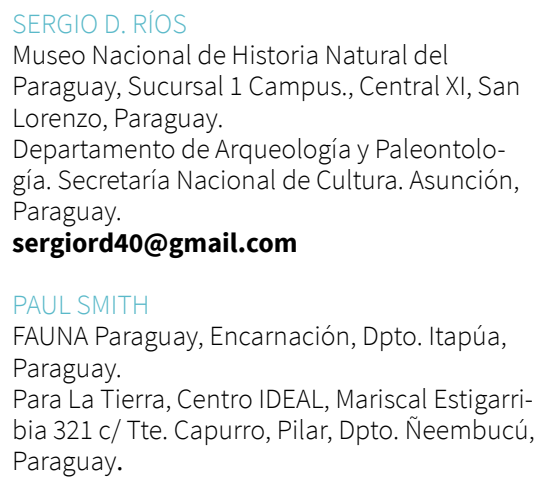

DOI: 10.32525/PARAQUARIANAT.2018(6):29.30 SSN 2309-5237
O Del/de los autor/es

Es con licencia exclusiva a Guyra Paraguay.

Recibido: 28 de diciembre de 2018
Aceptado: 11 de enero de 2019

Catacantha ferruginea (Draudt, 1929) es un pequeño satúrnido (Lepidoptera, Saturniidae) perteneciente a la subfamilia Hemleucinae. Su género incluye al menos once especies, todas de pequeño tamaño en relación a otras de la misma subfamilia (Brechlin et al. 2010). La especie $C$. ferruginea en ocasiones figura citada como perteneciente al género Perigopsis Bouvier, 1932, sinonimizado por Lemaire (2002). Se encuentra presente en el sur de Brasil (estados de Rio de Janeiro, São Paulo, Paraná y Santa Catarina) [Lemaire 2002, Siewert et al. 2010, Santos et al. 2015] y el noreste de Argentina (provincia de Misiones) [Nuñez Bustos 2015, Zapata et al. 2016], asociada claramente remanentes de Bosque Atlántico.

En esta nota presentamos el primer re porte de esta especie para el pais, Catacantha ferruginea, que a su vez constituye el primer hallazgo del géner $C$

Bouvier, 1930 en el Paraguay.

En fecha 24 de septiembre de 2018, horas de la mañana se halló un ejempla aparentemente recién muerto en una de las puertas de las casas de huéspedes de Mbaracayú Lodge, Jejui-Mi, departamento de Canindeyú (Figura 1). Material examinado: $1 \bigcirc$, Paraguay, Dpto. Canindeyú, R.N.B. Mbaracayu, 24.IX.2018, coll. P. Smith (CZPLT-i, Colección Entomológica Para La Tierra, Pilar).

\section{RESUMEN} Catacantha ferruginea (Draudt, 1929) en el Pa Reserva Natural Mbaracayú, departamento Canindeyú

PALABRAS CLAVE: biodiversidad, Bosque Atlántico, Canindeyú, polill
Se reporta el primer hallazgo del satúrnido

\section{ABSTRACT:}

The first Paraguayan record of the Saturnil (Draud, 1929) the Reserva Natural Mbaracayú, Canindeyú de partment.

KEY WORDS:
Canindeyú, moth.
Es posible asignar el material a C. ferruginea teniendo en cuenta la coloración geneal ocre que presenta. Comparte con C. ocuposee una coloración más amarillenta (Lemaire, 2002).

La presencia de esta especie en Paraguay no resulta sorpresiva, considerando la cercanía de los registros brasileños y argentAtlántico.

Es relevante mencionar que fuera del $s$ deste de Brasil, de donde se conoc rosos ejemplares (Miranda et al. 2015, Santos et al. 2015, Albertoni et al. 2018), se trat de una especie poco frecuente en las coleciones (Lemaire 2002). A su vez, constituy una evidencia de la diversidad entomológca aún desconocida presente en nuestros parques o reservas. A la fecha, no existe información del ciclo biologico o plantas huespedes de C. ferruginea (Lemaire 2002, Zapata et al. 2016), así que alentamos a que en nuestro país.

En años recientes se han sumado numerosos nuevos registros de la familia Saturniidae a la fauna del Paraguay (ej. Rios Diaz y Smith 2013, Smith et al. 2013,
Drechsel 2014a, Drechsel 2014b), lo que delata (Schaus, 1921) la presencia de ocelos

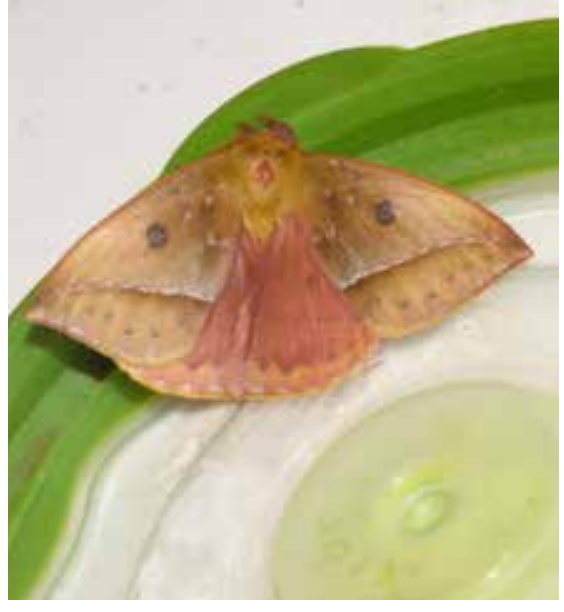

Figura 1. Macho de Catacantha ferruginea momento de ser colectado en la R.N.B. Mbaracayú, Paraguay.

muestra que dicha familia se conoce aún cion la adcion de Catacantha, son 20 los generos de Hemileucinae presentes en el Paraguay: $A u$ tomeris, Catacantha, Catharisa, Cerodirphia Dipha, Dirphopsis, Eubergia, Eudyaria, Gaia , Heliconisa, Hidripa, Hylesia, HyperchPerisa Periga, Pseudautomeris y Pseudodirphia (Lemaire 2002; Rios Diaz y Smith 2013, Ríos Diaz et al. 2016, Drechsel 2018). A modo de comparación, a la fecha hay 29 géneros de emileucinae reportados para Argentin (Núñez Bustos 2015) 